\title{
A Numerically Robust Sequential Linear Programming Algorithm for Reactive Power Optimization
}

\author{
Abraham Lomi ${ }^{1, *}$, Awan Uji Krismanto ${ }^{1}$, I Made Wartana ${ }^{1}$, and Dipu Sarkar ${ }^{2}$ \\ ${ }^{1}$ Department of Electrical Engineering, National Institute of Technology Malang, \\ Jl. Raya Karanglo, Km. 2, Malang 65143, Indonesia \\ ${ }^{2}$ Department of Electrical and Electronics Engineering, National Institute of Technology Nagaland, \\ Dimapur, Nagaland 797103, India
}

\begin{abstract}
A robust sequential primal-dual linear programming formulation for reactive power optimization is developed and discussed in this paper. The algorithm has the characteristic that no approximations or complicate control logic are required in the basic Sequential Linear Programming (SLP) formulation as used by other SLP algorithms reported in the literature. Transmission loss minimization is used as the primary objective. A secondary feasibility improvement objective is used which results in better feasible solution in comparison with the loss minimization objective especially when the initial base case has over voltages. Modification in the proposed method to obtain the limited amount and limited movement of controller solution for real time application is also presented. The algorithm has been tested on Ward and Hale 6-Bus system.
\end{abstract}

Keywords: Generator excitation, power flow, shunt reactive power, transformer taps, voltage stability.

\section{Introduction}

Proper reactive power dispatch is required for maintaining an acceptable level of the bus voltages, reduction in transmission losses, and an increase in static voltage stability margin. It is essential that existing reactive power controls viz., generator excitations, transformer taps, switchable shunt reactive power compensation are judiciously used to achieve the aforesaid objective. A new solution based on successive linear approximation has been used for power flow equations, and the quality of initial points regarding voltage magnitude is relatively low in the first few iterations [1]. An optimization method using Dynamical Thermal Rating (DTR) and linear programming (LP) to minimize generation costs or transmission losses derived from a spatially resolved thermal model of the transmission system based on actual weather conditions along the line [2]. A linear power flow model involving tap changers and phase shift considering transmission loss minimization is one of the common objective used in Linear Program (LP) formulations and implementation of expert system in solving the voltage stability with tap changers and generation controls

\footnotetext{
*Corresponding author: abraham@lecturer.itn.ac.id
} 
$[3,4]$. The following difficulties are encountered in the LP formulation with this minimization objective, (i) Zig-zagging in convergence characteristic of the sequential LP formulation, and (ii) Inability to remove over voltages with loss minimization objective.

To overcome the above difficulties and to avoid zig-zagging of the convergence characteristic, the authors in [3, 4] restricted the controller movements by using progressively smaller controller ranges in each power flow-LP optimization cycles. An efficient approach for solving the optimal reactive power dispatch problem with a nonlinear constraint optimization to find the control variable settings which minimize transmission active power losses and load bus voltage deviations [5]. Reference [6] presents a novel methods to approximate the nonlinear AC optimal power flow (OPF) into tractable linear/quadratic programming (LP/QP) based OPF problems that can be used for power system planning and operation. A development of a linear programming approach into a truly general purpose with computational of optimal power flow. A linearprogramming models that incorporate reactive power and voltage magnitudes in a linear power flow approximation has been presented [7].

In this paper a numerical robust sequential primal-dual sequential linear programming formulation for reactive power optimization is developed. The algorithm has the following features.

i). The algorithm does not require modified controller limits to control zig-zagging of the solution. Actual controller limits are used without any modification.

ii). The solution for the control variables is always within the specified limits and may be implemented directly for the power flow solution without any approximation.

iii). The number of power flow-optimization cycles are very small. Usually, an accurate minimum loss solution is obtained in two cycles to three cycles.

iv). Since modified controller ranges are not used, and controllers are allowed to move within their entire specified range, the number of controllers shifted from their initial position is small. When restricted control ranges are used as reported in other work $[3,4]$, the loss minimization is restricted due to insufficient control ranges. This results in activating more number of controller as well as more number of power flowoptimization cycles to achieve minimum transmission losses.

v). A secondary voltage feasibility improvement objective allows the algorithm to correct the over/under voltages efficiently. The transmission loss minimization objective is inefficient to correct the over voltages with standard LP formulation.

The implemented algorithm in a production grade program does not use any restriction on the magnitude of the floating point variables. Even the smallest possible pivot or the different possible floating point ratios computed in the primal-dual sequential linear programming algorithm are considered.

\section{The algorithm}

Standard LP formulation solves an optimization problem either as maximization or as minimization problem. The minimization problem is a dual of the maximization problem and essentially gives the same optimum results as the maximization problem. The LP algorithm for the maximization problem is the primal (simplex) algorithm and for the minimization problem is the dual (dual simplex) algorithm. A primal algorithm requires a sub optimal but feasible tableau. A dual algorithm requires optimal tableau with infeasibilities [8]. A primal pivot improves the feasibility while attempting to maintain optimality. When the initial tableau is neither optimal nor feasible a straight forward implementation of the primal or the dual algorithm is not possible. Under these conditions a primal-dual algorithm may be used. A primal and dual pivots in terms of their influence on the objective and accordingly selects either the primal or dual pivot. The following basic 
difference between the primal and the dual algorithm is of importance to the transmission loss minimization problem.

i) When the initial tableau is upper bound feasible, a primal algorithm will always provide feasible controller solutions that may be implemented directly for the subsequent power flow solution. Further, the lower bound infeasibilities, such as low voltage will improve with the improvement in the objective. Hence, there is no need to use restricted or modified controller ranges as used in other reported works.

ii) There is no guarantee that a straightforward implementation of the dual algorithm will result in feasible controller solutions. This appears to be the main reason for the use of the approximations on controller limits as reported in the earlier literature. In the primal-dual algorithm presented in this paper a check is introduced to see whether a given pivot will result in infeasible controller solution. If so, this particular pivot is discarded and the next possible pivot is considered. This ensures that the final solution for the controller variables will always be within the specified range.

\section{Problem statement}

The transmission loss minimization problem may be stated as follows, in Equation (1) to Equation (5).

$$
\begin{aligned}
& \text { Minimize } f(x, u) \\
& \text { Subject to } g(x, u)=0 \\
& u_{\min } \leq u \leq u_{\max } \\
& x_{\min } \leq x \leq x_{\max } \\
& h_{\min } \leq h \leq h_{\max }
\end{aligned}
$$

The power flow equation constraints defined in Equation (2) to be satisfied at any operating point. The vectors $u$ and $x$ represent set of control variables (generator excitations, transformer taps etc.) and dependent variables (bus voltage magnitudes). Constraints defined in Equation (3) and Equation (4) are dependent variable permissible control limits. Constraints defined in Equation (5) are security constraints with the limitation of MVAR loading of generators and MVA loading of transmission lines in the system.

The algorithm presented in this paper minimizes active power of the slack generator. This is equivalent to transmission loss minimization, when the active power generations of the remaining generators are determined from economic dispatch.

\section{Reduced formulation}

Linearizing the power flow equations around its solution [9], it describes in Equation (6) to Equation (8) that is obtained:

$$
\begin{aligned}
& {\left[\frac{\partial g}{\partial x}\right] \Delta x+\left[\frac{\partial g}{\partial u}\right] \Delta u=0} \\
& \Delta x=-\left[\frac{\partial g}{\partial x}\right]^{-1}\left[\frac{\partial g}{\partial u}\right] \Delta u \\
& \Delta x=-\left[S_{x}\right] \Delta u
\end{aligned}
$$

Equation (8) gives the sensitivity of dependent bus voltage magnitude and phase angles as a function of specified control variables, in Equation (9). 


$$
\begin{gathered}
\Delta P_{s l}=\left[\frac{\partial P_{s l}}{\partial x}\right]^{T} \Delta x+\left[\frac{\partial P_{s l}}{\partial u}\right]^{T} \Delta u \\
\quad=\left(\left[\frac{\partial P_{s l}}{\partial x}\right]^{T}\left[S_{x}\right]+\left[\frac{\partial P_{s l}}{\partial u}\right]^{T}\right) \Delta u
\end{gathered}
$$

Equation (9) gives the sensitivity of slack generation as a function of the specified variables (Equations 10 and 11).

$$
\begin{aligned}
\Delta h= & {\left[\frac{\partial h}{\partial x}\right] \Delta x+\left[\frac{\partial h}{\partial u}\right] \Delta u } \\
& =\left(\left[\frac{\partial h}{\partial x}\right]\left[S_{x}\right]+\left[\frac{\partial h}{\partial u}\right]\right) \Delta u
\end{aligned}
$$

Equation (11) gives the sensitivity of system security monitoring variables such as generator reactive power limits, line loading as functions of specified control variables.

\subsection{Simplex tableau formulation}

The transmission loss minimization LP problem [10] can be stated in Equation (12) to Equation (16).

$$
\begin{array}{cc}
\text { Minimize } & \Delta P_{s l}=C^{T} \Delta u \\
\text { Subject to } & {\left[S_{x}\right] \Delta u \geq \Delta x_{\min }} \\
-\left[S_{x}\right] \Delta u \geq-\Delta x_{\max } \\
\Delta u=\Delta u_{\min } \\
-\Delta u=-\Delta u_{\max }
\end{array}
$$

where Equation (13) and Equation (14) include the linearized sensitivity relations Equation (7) and Equation (11). In the actual implementation, negative of the objective function (12) is maximized and the sign of the inequalities in Equation (13) to Equation (16) is reversed. With these modifications a condensed simplex tableau can be readily formed and is shown in the tableau Equation (17),

$$
\begin{array}{|c|c|}
\hline-S x & -\Delta x_{\min } \\
S x & \Delta x_{\max } \\
-I & -\Delta u_{\min } \\
I & \Delta u_{\max } \\
\hline C^{\mathrm{T}} & 0.0 \\
\hline
\end{array}
$$

Where $r$ is a column vector representing the negative of the right hand side of the inequalities Equation (13) to Equation (16) and $A$ is the coefficient matrix of control variables representing the negative of the left-hand side of the inequalities Equation (13) to Equation (16). 


\subsection{Sensitivities}

\subsubsection{Sensitivities with respect to dependent bus voltage magnitudes and angles}

To obtain the sensitivities of the injection buses with respect to the voltages magnitude and angles of the dependent bus $(|V|, \delta)$ and the voltage magnitude of the independent bus (generator excitations), a partial derivatives in the formulation of the Jacobian power flow are used Equation [11]. The following terms Equation (18) to Equation (20) are used in the partial derivatives.

$$
\begin{aligned}
Y_{k m} & =\left(G_{k m} j B_{k m}\right) \\
E_{m} & =\left(e_{m}+j f_{m}\right) \\
I_{m} & =\left(a_{m}+j b_{m}\right)
\end{aligned}
$$

The partial derivatives when $k \neq m$ are given by Equation (21) and Equation (22)

$$
\begin{aligned}
& \frac{\partial P_{k}}{\partial \delta_{m}}=\frac{\partial Q_{k}}{\partial\left|E_{m}\right|}\left|E_{m}\right|=a_{m} f_{k}-b_{m} e_{k} \\
& \frac{\partial P_{k}}{\partial\left|E_{m}\right|}\left|E_{m}\right|=-\frac{\partial Q_{k}}{\partial \delta_{m}}=a_{m} e_{k}+b_{m} f_{k}
\end{aligned}
$$

The partial derivatives when $k=m$ are given by Equation (23) to Equation (26)

$$
\begin{aligned}
& \frac{\partial P_{k}}{\partial \delta_{m}}=-Q_{k}-B_{k k}\left|E_{k}\right|^{2} \\
& \frac{\partial Q_{k}}{\partial\left|E_{k}\right|}=Q_{k}-B_{k k}\left|E_{k}\right|^{2} \\
& \frac{\partial P_{k}}{\partial\left|E_{k}\right|}\left|E_{k}\right|=P_{k}+G_{k k}\left|E_{k}\right|^{2} \\
& \frac{\partial Q_{k}}{\partial \delta_{k}}=P_{k}-G_{k k}\left|E_{k}\right|^{2}
\end{aligned}
$$

The sensitivities is ignored with respect to the slack bus.

\subsubsection{Sensitivities with respect to shunt reactive power compensation}

If $B_{\mathrm{sh}}$ and $V_{\mathrm{sh}}$ are defined as the reactive power compensation susceptance and the voltage at a bus respectively, the reactive power absorbed by the shunt component is given by Equation (27)

$$
Q_{s h}=-\left|V_{s h}\right|^{2} B_{s h}
$$

The reactive power absorption sensitivity as a function of shunt susceptance is given by Equation (28)

$$
\frac{\partial Q_{s h}}{\partial B_{s h}}=-\left|V_{s h}\right|^{2}
$$

The right-hand side of Equation (28) is -1.0 for constant power compensation. 


\subsubsection{Sensitivities with respect to transformer tap}

Let $p$ and $q$ are defined as the transformer terminal buses with the off nominal turn ratio $T: 1$. With the relation $T=1 / \tau$, the sensitivities of the transformer power flow with respect to the transformer tap are given by the following Equations (29) to Equation (32):

$$
\begin{aligned}
& \frac{\partial P_{p q}}{\partial \tau}=2 \pi\left|V_{p}\right|^{2} G_{p q}-\left|V_{p} \| V_{q}\right|\left|y_{p q}\right| \cos \left(\delta_{p}-\delta_{q}-\theta_{p q}\right) \\
& \frac{\partial Q_{p q}}{\partial \tau}=-2 \pi\left|V_{p}\right|^{2} B_{p q}-\left|V_{p} \| V_{q}\right|\left|y_{p q}\right| \sin \left(\delta_{p}-\delta_{q}-\theta_{p q}\right) \\
& \frac{\partial P_{q p}}{\partial \tau}=-\left|V_{p}\right|\left|V_{q}\right|\left|y_{p q}\right| \cos \left(\delta_{p}-\delta_{q}-\theta_{p q}\right) \\
& \frac{\partial Q_{q p}}{\partial \tau}=-\left|V_{p} \| V_{q}\right|\left|y_{p q}\right| \sin \left(\delta_{p}-\delta_{q}-\theta_{p q}\right)
\end{aligned}
$$

Where $y_{p q}=G_{p q}+j B_{p q}$, is the series admittance between the buses $p$ and $q . \theta_{p q}$ is the phase angle of $y_{p q}$.

\subsection{Primal-dual algorithm}

The pivot selection in the primal - dual algorithm is explained with reference to Equation (17). Let $A(p, q)$ represents the pivot. $r(p)$ is the corresponding entry in the vector $r$ and $C(q)$ is the corresponding entry in the objective row. Then a primal pivot must satisfy the following conditions. First, $C(q)$ is the most negative entry in the objective row. Second, The ratio $r(p) / A(p, q)$ is the smallest positive ratio for all possible pivots in column $q$.

A dual pivot must satisfy the following conditions: i) $r(p)$ is the most violated basis variable. ii) The ratio $-(C(q) / A(p, q))$ is the smallest positive ratio among all possible pivots in row $p$. Once a primal and a dual pivot are found, whichever pivot influences the objective most is chosen as the pivot.

\subsubsection{Implementation}

In the actual implementation the following two restrictions are added.

i). The pivot should not result in any control infeasibility.

ii). After pivoting, a new tableau obtained and more feasible compare the previous one.

This requires the simulation of the effect of the pivot on the vector $r$.

The first condition is always satisfied with a primal pivot, provided that the initial state has feasible controller positions. A dual pivot does not necessarily satisfy the two restrictions stated above. There is no guarantee that it will result in feasible controller solution. Hence a check is required to ensure the same. Although the dual pivot forces the most violated variable to its limit, there is no guarantee that the overall feasibility of the tableau improves. When the two restrictions stated above are implemented, it is guaranteed that the algorithm will result in implementable solution for the control variables with improved optimality feasibility. For practical large systems, the final tableau will be usually optimal with some infeasibility. 


\section{Test case and results}

The proposed algorithm is tested on Ward and Hale 6 Bus system. This case system is taken from reference [12]. The sensitivity matrix $S_{x}$ is shown in Table 1 . The sensitivity information is obtained from coupled load flow Jacobian formulation.

The first two columns correspond to generator excitation controls $\left|V_{1}\right|$ and $\left|V_{2}\right|$. The next two columns correspond to the shunt reactive power controls at buses 4 and 6 . The last two columns correspond to the transformer tap controls. The first two rows of Table 1 correspond to the generator reactive power sensitivity with respect to the specified controllers.

The last four rows give the sensitivity of the dependent bus voltages (magnitudes) or the buses 3 to 6 concerning the specified controllers. The convergence characteristic of the algorithm is listed in Table 2. The last column of the tableau $\left(S_{v}\right)$ represents the absolute sum of voltage infeasibilities. Accurate convergence is obtained in two load flow optimization cycles. Further improvement in the loss reduction was not possible since two of the bus voltages reached upper bound limits. The algorithm does not experience any oscillations with further power flow optimization cycles.

Table 1. Calculated parameters of ward-hale 6 bus system

\begin{tabular}{|c|c|c|c|c|c|}
\hline$\left|V_{1}\right|$ & $\left|V_{2}\right|$ & $Q C_{4}$ & $Q C_{6}$ & Tap 6-5 & Tap 4-3 \\
\hline $.84875 \mathrm{E}+00$ & $-.17305 \mathrm{E}+01$ & $-.92173 \mathrm{E}+00$ & $-.86877 \mathrm{E}+00$ & $.71902 \mathrm{E}+00$ & $.43818 \mathrm{E}+00$ \\
$-.16784 \mathrm{E}+01$ & $.15604 \mathrm{E}+01$ & $-.32603 \mathrm{E}+00$ & $-.40574 \mathrm{E}+00$ & $-.79242 \mathrm{E}+0$ & $-.60662 \mathrm{E}+00$ \\
$.75541 \mathrm{E}+00$ & $.40950 \mathrm{E}+00$ & $.20617 \mathrm{E}+00$ & $.10027 \mathrm{E}+00$ & $-.96008 \mathrm{E}-01$ & $.79354 \mathrm{E}+00$ \\
$.90441 \mathrm{E}+00$ & $.33739 \mathrm{E}+00$ & $.24566 \mathrm{E}+00$ & $.12116 \mathrm{E}+00$ & $-.10332 \mathrm{E}+0$ & $-.11531 \mathrm{E}+00$ \\
$.58742 \mathrm{E}+00$ & $.66880 \mathrm{E}+00$ & $.81089 \mathrm{E}-01$ & $.18831 \mathrm{E}+00$ & $.52592 \mathrm{E}+00$ & $-.69034 \mathrm{E}-01$ \\
$.85948 \mathrm{E}+00$ & $.40240 \mathrm{E}+00$ & $.12223 \mathrm{E}+00$ & $.27130 \mathrm{E}+00$ & $-.21841 \mathrm{E}+0$ & $-.58726 \mathrm{E}-01$ \\
\hline
\end{tabular}

Table 2. Parameter performed of Ward-Hale 6 Bus system.

\begin{tabular}{|c|c|c|c|}
\hline $\begin{array}{c}\text { Optimization } \\
\text { Cycle No. }\end{array}$ & Loss (MW) & $\begin{array}{c}\text { Compensation } \\
\text { (MVAR) }\end{array}$ & $\mathrm{S}_{\mathrm{v}}(\mathrm{p} . \mathrm{u})$ \\
\hline 0 & 11.612 & 00.000 & 0.045 \\
1 & 9.283 & 10.500 & 0.000 \\
2 & 9.200 & 10.500 & 0.000 \\
\hline \multicolumn{3}{|c|}{$S_{v}$} \\
\hline
\end{tabular}

Table 3. Parameter optimization of ward-hale 6 bus system.

\begin{tabular}{|c|c|c|}
\hline Control & Initial & Final \\
\hline$\left|V_{1}\right|$ (p.u) & 1.0500 & 1.1000 \\
$\left|V_{2}\right|$ (p.u) & 1.1000 & 1.1070 \\
TAP 6-5 & 1.0250 & 0.9125 \\
TAP 4-3 & 1.1000 & 0.9625 \\
MVAR at bus 4 & 0.0000 & 5.0000 \\
MVAR at bus 6 & 0.0000 & 5.5000 \\
\hline
\end{tabular}




\section{Conclusion}

A numerically robust primal-dual sequential LP algorithm for transmission loss minimization is presented in this paper. The algorithm does not use any approximations on the controller limits or intricate control logic as suggested by other previous algorithms presented in the literature.

The algorithm has excellent convergence characteristics towards minimum losses with improved feasibility. Accurate minimum loss solutions were obtained from point 2 and 3 in LP load flow cycles. While minimizing losses, overvoltages are seldom introduced.

For practical large scale systems, only marginal over voltages were present at the point of convergence. The algorithm has the basic characteristic of curtailing significant number of controller movement. Modification to the basic algorithm to reduce the number of controllers are easier and straight forward to implement. The algorithm strictly respects any specified ranges for the control variables movement to any desired degree by specifying appropriate controller ranges. The algorithm, when used with a secondary feasibility improvement objective, results in better loss reduction with improved feasibility. Further over voltages are effectively removed by the algorithm. Operator's control priorities may be very easily incorporated in the algorithm, while arriving at the effective subset of the controllers.

The authors would like to thank the Directorate General of Research and Development Strengthen c.q. Directorate of Research and Community Service, Ministry of Research, Technology, and Higher Education, Government of the Republic of Indonesia for financial support for multiyear research scheme (2019-2021) under the contract Number: 7/E/KPT/2019.

\section{References}

1. Z. Yang, H. Zhong, Q. Xia, A. Bose, C. Kang, IET Gener. Transm. Dis, 10,14:36543662(2016). https://doi.org/10.1049/iet-gtd.2016.0547

2. M. Khaki, P. Musilek, J. Heckenbergerova, D. Koval, Electric power system cost/loss optimization using dynamic thermal rating and linear programming. 2010 IEEE Electrical Power \& Energy Conference Halifax, NS, Canada, 2010). p. 1-6. https://doi.org/10.1109/EPEC.2010.5697195

3. A. Lomi, D. Thukaram, Telkomnika, 10,2:257-264(2012). http://dx.doi.org/10.12928/telkomnika.v10i2.793

4. A. Lomi, F. Y. Limpraptono, JTEC, 10(2-3):13. (2018). https://journal.utem.edu.my/index.php/jtec/issue/view/189

5. Z. Li, J. Yu, Q.H. Wu, IEEE T. Power Syst., 33,4:4593-4603(2017). https://doi.org/10.1109/TPWRS.2017.2776253

6. P. Fortenbacher, T. Demiray, J. Electr. Power Energy Syst., 10:680-689(2019). https://doi.org/10.1016/j.ijepes.2018.12.008

7. J.A. Castrillon, J.S. Giraldo, C.A. Castro, Mixed integer linear programming formulation for optimal reactive compensation and voltage control of distribution power systems, IEEE Power \& Energy Society General Meeting, 2017. (Chicago, IL, USA, 2017). p. 1-5. https://doi.org/10.1109/PESGM.2017.8273929

8. R.S. Ferreira, C.L.T. Borges, M.V. Pereira, IEEE T. Power Syst., 29:524472459(2014). https://doi.org/10.1109/TPWRS.2014.2304539

9. T. Sowa, A. Stroband, W. Cramer, S. Koopmann, A. Schnettler, An AC Power flow linearization for power system optimization using linear programming, IEEE 
Electrical Power and Energy Conference (EPEC), IEEE Xplore, (Ottawa, ON, Canada, 2016), p. 1-5. https://doi.org/10.1109/EPEC.2016.7771674

10. S. Mhanna, G. Verbič and A. C. Chapman, Tight linear programming approximations for the optimal problem, Power System Computation Conference, IEEE Xplore, (Genoa, Italy, 2016), p. 1-7. https://doi.org/10.1109/PSCC.2016.7540937

11. J.R. Martí, H. Ahmadi, L. Bashualdo, IEEE T. Power Syst., 28,3:1682-1690(2013). https://doi.org/10.1109/TPWRD.2013.2247068

12. N.T. Rao, A. Jagannadham, Optimal reactive power flow control for minimization of active power losses using Particle swarm Optimization. 2015 Conference on Power, Control, Communication and Computational Technologies for Sustainable Growth (PCCCTSG) (Kurnool, India, 2015), p. 38-41.

https://doi.org/10.1109/PCCCTSG.2015.7503954 